\title{
Absence of the Septum Pellucidum
}

National Institute of Neurological Disorders and Stroke (NINDS)

\section{Source}

National Institute of Neurological Disorders and Stroke (NINDS). Absence of the Septum

Pellucidum Information Page.

The septum pellucidum (SP) is a thin membrane located at the midline of the brain between the two cerebral hemispheres, or halves of the brain. It is connected to the corpus callosum -- a collection of nerve fibers that connect the cerebral hemispheres. This rare abnormality accompanies various malformations of the brain that affect intelligence, behavior, and the neurodevelopmental process, and seizures may occur. Children who are born without this membrane and also have other abnormalities-pituitary deficiencies and abnormal development of the optic disk--have a disorder known as septo-optic dysplasia. 